\title{
Editorial for Special Issue on Social Computing
}

\author{
Deze Zeng ${ }^{1} \cdot$ Song $\mathrm{Guo}^{2} \cdot \mathrm{Bo} \mathrm{Li}^{3}$
}

Published online: 3 August 2016

(C) Springer Science+Business Media New York 2016

\section{Editorial:}

With the rapid development of mobile computing technologies, social computing emerges as a new computing paradigm that enables people to quickly and easily participate in social interactions, contribute their knowledge and share usergenerated content, experiences and opinions. Social computing provides a wide platform where information flows elegantly according to people's demands. Social computing is empowered by various technologies across different disciplines, such as crowdsourcing, collective intelligence, peerto-peer computing, wireless access, device-to-device (D2D) communications and cloud computing, which jointly build a solid foundation to support social computing. On the one hand, these technologies evolve individually in their respective sectors to advance the development of social computing. On the other hand, they are also inversely affected by the requirements from social computing. The proliferation of social computing imposes many new challenges to these related areas. For example, the high distribution nature of social computing first requires a scalable and elastic infrastructure to meet both the communication and computation requirements. The social relationship inherent in social computing also has a deep impact on the computation, communication and storage

Deze Zeng

deze@cug.edu.cn

1 School of Computer Science, China University of Geosciences, Wuhan, Hubei, China

2 School of Computer Science and Engineering, The University of Aizu, Aizuwakamatsu, Fukushima, Japan

3 Department of Computer Science and Engineering, Hong Kong University of Science and Technology, Clear Water Bay, Kowloon, Hong Kong resource management. Therefore, it is significant to jointly investigate social computing and its related disciplines to address these challenges for the progress of social computing.

This special issue features six selected high-quality papers covering a variety of aspects related to social computing, including service provision, resource allocation and management, network control, security and privacy issues.

The first article "Cost-Effective Service Provisioning for Hybrid Cloud Applications" identifies how to use hybrid cloud to provision social services in a cost-effective manner, with the consideration of load balancing, cloud computing platforms dynamics (e.g., breakdown or update) and price changes. The authors (Fangming Liu, Bin Luo and Yipei Niu) propose a Least Cost per Connection (LCC) algorithm to distribute job requests to the most cost-effective clouds while adapting changes among multiple public clouds. Compared with single cloud strategy, the proposed strategy decreases the cost and latency by $30.2 \%$ and $10.1 \%$, respectively.

The second article titled "UFalloc: Towards Utility Maxmin Fairness of Bandwidth Allocation for Applications in Datacenter Networks" by Fei Xu, Wangying Ye, Yuhan Liu and Wei Zhang then discusses bandwidth allocation for cloud applications in sharing datacenter networks. They notice that existing bandwidth allocation solutions may fail to guarantee the fairness of application performance (e.g., job completion time or throughput). To solve this problem, the authors propose a rigorous definition of application-level utility max-min fairness and then design an application-level Utility max-min Fair bandwidth allocation strategy called UFalloc. They also implement UFalloc in OpenFlow controller and evaluate its performance via Mininet simulator. The results show that UFalloc can improve the network utilization while achieving a tunable max-main fairness.

The third article "Social-aware Computing based Congestion Control in Delay Tolerant Networks" applies the 
social characteristics to tackle the congestion control problem in Delay Tolerant Networks (DTNs). The authors (Yan Liu, Kun Wang, Huang Guo, Qing Lu and Yanfei Sun) define a social congestion metric (SCM) to measure the congestion level of a node with the consideration of social features. By exploring such metric, they further propose a message forwarding strategy, in which messages are always forwarded to the nodes with higher SCM. At the same time, a message dropping mechanism that drops the message with minimum social links is also invented. Simulation based studies using ONE simulator and Helsinki Map are conducted to show the high efficiency of the proposal.

In the fourth article "Automatically Setting ParameterExchanging Interval for Deep Learning", the authors Siyuan Wang, Xiaofei Liao, Xuepeng Fan, Hai Jin, Qiongjie Yao and Yu Zhang notice that parameter exchanging between the master and slaves in parameter-server frameworks imposes a bottleneck to the performance of deep learning algorithms as around $80 \%$ of the time may be consumed by parameter exchange according to their experiment results. They further conjecture that parameter exchanging interval setting plays a critical role. To this end, they propose an automatic exchanging interval setting approach with the awareness of the master available resources to avoid request bursts. Experiment results show an 8-time speedup compared with existing parallel stochastic gradient descent (SGD) algorithm.

The fifth article "A Survey on Security in D2D Communications" by Mingjun Wang and Zheng Yan provides a survey on security in D2D communications. In this article, they first explore a D2D security architecture, investigate its security threats and specify security requirements accordingly. The authors then further review some existing solutions on D2D security in the literature and compare their advantages and disadvantages. Based on the understanding of existing related work, the authors finally outline open research challenging issues.

The last article with the title "Cryptanalysis on a SecretSharing Based Conditional Proxy Re-Encryption Scheme" by Yuxia Sun and Dan Liu makes a cryptanalysis on a conditional proxy re-encryption scheme that supports "AND" and "OR" gates over multiple conditions. Although the scheme is declared to be secure against chosen-ciphertext attack (CCA) in the random oracle model, they show that the scheme has potential security weakness and therefore is not CCA secure by proposing two attack models. The authors also analyze the reasons and further highlight the security risks that shall be prevented to enable secure encrypted-data-sharing with multiple keyword-based conditions.

Finally, taking this opportunity, the guest editors would like to thank all the authors who submitted their work to this special issue and express our sincere appreciation to all the reviewers for their efforts in reviewing the manuscripts.

Deze Zeng received his Ph.D. and M.S. degrees in computer science from University of Aizu, Aizu-Wakamatsu, Japan, in 2013 and 2009, respectively. He received his B.S. degree from School of Computer Science and Technology, Huazhong University of Science and Technology, China in 2007. He is currently an associate professor in School of Computer Science, China University of Geosciences, Wuhan, China. His current research interests include: network function virtualization, cloud computing, software-defined networking, wireless sensor networks, data center networking, networking protocol design and analysis. He has authored 1 book and over 70 papers in refereed journals and conferences in these areas. He is a member of IEEE.

Song Guo received the $\mathrm{PhD}$ degree in computer science from the University of Ottawa, Canada. He is currently a full professor at the University of Aizu, Japan. His research interests are mainly in the areas of wireless network, cloud computing, big data, and cyber-physical system. He has authored/edited 7 books and over 300 papers in refereed journals and conferences in these areas. He serves/served in editorial boards of IEEE Transactions on Parallel and Distributed Systems, IEEE Transactions on Emerging Topics in Computing, IEEE Communications Magazine, Wireless Networks, Wireless Communications and Mobile Computing, and many other major journals. He has been the general/ program chair or in organizing committees of numerous international conferences. Dr. Guo is a senior member of IEEE, a senior member of ACM, and an IEEE Communications Society Distinguished Lecturer.

Bo Li is a professor in the Department of Computer Science and Engineering. Hong Kong University of Science and Technology. He was a Cheung Kong Chair Professor in Shanghai Jiao Tong University (2010-2013) and an adjunct researcher in Microsoft Research Asia (1999-2007) and in Microsoft Advance Technology Center (20072009). His current research interests include: multimedia communications, large-scale content distribution in the Internet, datacenter networking, cloud computing, and wireless sensor networks. He is a Fellow of IEEE. He has been an editor or a guest editor for a dozen of ACM and IEEE journals and magazines. He was the Co-TPC Chair for IEEE INFOCOM 2004. He received his B. Eng. Degree in the Computer Science from Tsinghua University, Beijing, and his Ph.D. degree in the Electrical and Computer Engineering from University of Massachusetts at Amherst. 